\title{
Treatment Options for Erectile Dysfunction in Patients Failing Oral DrugTherapy
}

\author{
Konstantinos Hatzimouratidis, Dimitrios G. Hatzichristou* \\ 2nd Department of Urology, "Papageorgiou” General Hospital, Aristotle University of Thessaloniki, Thessaloniki, Greece
}

\begin{abstract}
Oral drug therapy is the first line treatment for erectile dysfunction. Efficacy of oral drugs does not exceed $70 \%$, while drop-out rate is high. Therefore, salvage strategies and second/third line treatment options are necessary to restore erectile function in such patients. The article reviews currently available data and comment on key issues that urologists address on their every day clinical practice.

In order to do so, a literature-based critical presentation on the current methodological problems on definition of treatment failure, treatment outcome assessment and alternative therapeutic options was made.

Emphasis was given in identifying truly non-responders, as proper instructions for oral drug administration and psychosexual counselling may reverse a substantial number of non-responders to responders. Medication, clinical and patient/partner related issues have been identify that may lead to treatment failure. Treatment outcome assessment based not only on quality of erectile response, but also on side effects profile and patient satisfaction is recommended. Treatment options for truly non-responders include intracavernosal injections, combination therapies, and penile prosthesis. Management strategy however, must identify patient and partner needs and expectations and involve them in the decision-making.
\end{abstract}

(C) 2004 Elsevier B.V. All rights reserved.

Keywords: Penile erection; Drug therapy; Penis; Pharmacology; Treatment failure

\section{Introduction}

In the past 20 years, erectile dysfunction became one of the major fields in medical research. The understanding of the pathophysiology of the disease has expanded. The enormous scientific data in basic and clinical research led to the development of numerous treatment options. However, the introduction of sildenafil, a specific phoshodiesterase 5 inhibitor in 1998, as the first oral drug with proven efficacy and safety in the treatment of erectile dysfunction has changed dramatically the management of our patients [1]. Sildenafil prescriptions raised significantly while the use of intracavernosal injections and intraurethral alprostadil - that were the only approved pharmacotherapiesremained unchanged [2]. Later apomorphine-a central acting dopaminergic agonist-and last year, two

\footnotetext{
* Corresponding author. Tel. +30-231-0285990;

Fax: +30-231-0263939.

E-mail address: hatzichr@med.auth.gr (D.G. Hatzichristou).
}

new phosphodiesterase 5 inhibitors-tadalafil and vardenafil-expanded treatment options. The availability of oral drugs received wide publicity and numerous patients seek treatment for erectile dysfunction.

The efficacy of phosphodiesterase inhibitors in almost all published clinical trials is about $70 \%$ [3-5]. However, in specific groups of patients (e.g. diabetes mellitus, radical prostatectomy) the efficacy is significantly lower. This means that at least $30 \%$ of men will not respond well to oral agents. As a result, a significant group of patients will need some other form of intervention to make them sexually functional. In many countries the majority of patients with erectile dysfunction are treated in primary care settings. The management of ED by non-specialists includes minimal patient work-up and prescription of exclusively first line treatment options in most cases, mainly due to the lack of time and background knowledge [6]. Treatment failures are then referred to specialists [7]. So the role of the urologist in treating these failures is expanding and today he has to deal with more and more 


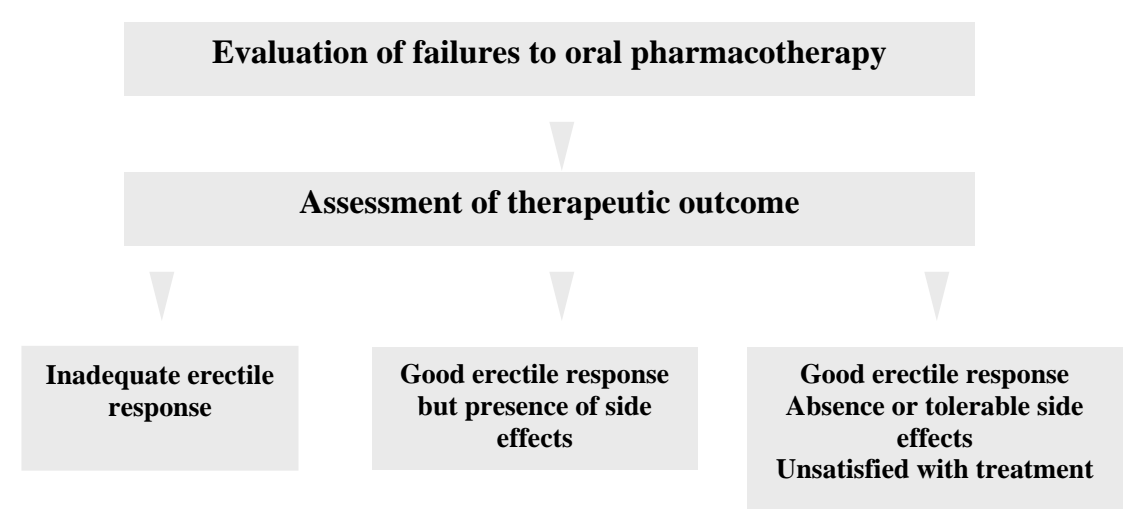

Fig. 1. Evaluation of failures to oral pharmacotherapy.

patients who do not respond adequately to oral pharmacotherapy. Treatment strategy for these patients is therefore urgently needed.

\section{Treatment outcome assessment}

Assessment of treatment outcome in patients with erectile dysfunction who failed oral drug pharmacotherapy is a multifactorial process. The quality of erectile response is not the only aspect in these patients but side effects and patient satisfaction from treatment are two other crucial factors that have to be carefully addressed (Fig. 1).

Erectile response may be graded as complete, partial or no response. The successful treatment of erectile dysfunction is associated with rigid and sustaining erection till ejaculation in most of attempts (complete response). In patients who failed oral treatment, there is no erectile response or the response may be partial. A partial erectile response may be either a full erection but without adequate rigidity and good sustaining capability till ejaculation, or a rigid but not always maintaining erection (detumescence before ejaculation). Patients with no response experience either inadequate rigidity for vaginal penetration or a rigid erection but most of the times not maintaining for completion.

Most of the side effects with oral pills for ED are rare, mild and well tolerated. However, if present, they also may also be graded as completely lacking, tolerable or non-tolerable. Some patients experience moderate side effects that can be tolerated by adapting a certain way of administration (e.g. use of pain relief drugs, drug administration skipping meals -due to dyspepsia). These patients need to be reassured about side effects and advised that side effects may be selflimited after continuing treatment for a few more attempts. Finally, a few patients experience severe, non tolerated side effects that cause discontinuation of the treatment. In the presence of side effects patients should consider alternative oral treatment options.

Assessment of satisfaction is also mandatory. Erectile dysfunction is not a life-threatening disease and patients need to be satisfied from their sexual life and not just having a successful intercourse. When a patient alone or, in case of existing relationship, patient and his partner are satisfied, the term complete satisfaction is used. Some patients or their partners may be happy with the erectile response but not happy using the pill (dependency on the drug, scheduling, interpersonal problems). These patients experience partial satisfaction and they are in high risk for drop-out. On the other hand, patients may not be satisfied with their erectile response, or they may experience side effects or may have relationship problems and/or partner reluctance to treatment; such patients will never use the pill.

\section{Definition of treatment failure to oral drugs}

Although the term 'treatment failure' to oral drugs is widely used, there is no precise definition of what exactly means. Based on the definition of erectile dysfunction, treatment failure of a drug means that the medication does not result in an erection that is sufficient for vaginal penetration or the erection does not last enough to complete the intercourse. The disability to attain and/or maintain an erection may be permanent (in all attempts for intercourse) or occasional.

Treatment failure may be due to medication, clinician and patient issues (Table 1). Medication issues include the efficacy rates in published clinical trials which are about $70 \%$. Several subgroups of patients with erectile dysfunction experience significantly 
Table 1

Factors leading to an inadequate trial of oral pharmacotherapy

\begin{tabular}{|c|c|c|}
\hline Medication issues & Clinician issues & Patient/partner issues \\
\hline Efficacy rates and treatment reliability & Inadequate administration instructions & Unrealistic expectations \\
\hline Special populations reflect variable responses & Inappropriate dosing & Lack of persistence \\
\hline (difficult-to-treat subgroups) & Misdiagnosis & Fear/reluctance to treatment \\
\hline Side effects profile & Lack of follow-up & $\begin{array}{l}\text { Unaddressed psychological issues } \\
\text { Partner issues }\end{array}$ \\
\hline
\end{tabular}

lower efficacy rates as the diabetes mellitus and the post-radical prostatectomy subgroups. Medication issues also include side effects as mentioned previously.

Clinician issues include inadequate instructions or dosing of the drug. Several data show that common failures to sildenafil may be due to insufficient sexual stimulation before attempting intercourse, intake of the drug after a full meal, lack of titration to the maximum tolerated dose and lack of adjustment of dosing.

All oral pills are facilitator of erection; they do not initiate an erection [8]. Sexual stimulation is therefore essential; on the other hand the time of onset although may be even 15-20 min in some cases, the vast majority of the patients recognize the best effect at least 1 hour after administration. Food interactions and delayed drug absorption and low plasma concentration after especially fatty meals have been described with sildenafil and vardenafil. Titration to the maximum tolerated dose is essential, as marketing experience has shown that more than $80 \%$ of the patients prefer the higher dose due to enhanced efficacy. One of the most common causes of lack of titration is the fear for side effects. Adjustment of dosing is also necessary. It is considered that at least 4 sexual attempts with the maximum recommended dose of the drug in order to overcome treatment anxiety and suboptimal sexual stimulation are necessary to characterize treatment failure. Based on the above data definition of a nonresponder to oral pharmacotherapy may be as follows: an inadequate erectile response after at least 4 attempts of the highest tolerated drug dose in accordance with manufacturer's guidelines with respect to timing relative to meals, alcohol ingestion, use of concomitant medications, and adequate sexual stimulation/arousal.

Besides these reasons for inappropriate intake of sildenafil, one of the most common causes of discontinuation of a drug that is considered as treatment failure is the lack of follow-up of the patients. Erectile dysfunction is a chronic disease. Follow-up visits are essential to improve physician-patient communication, solve treatment problems that may have occurred, identify any change in potency status or new medical conditions and offer continuing education to patients and their partners. These essential aspects in managing ED patients can be easily remembered by the acronym FAST (Follow-up of patients, Adjustment of time of administration, Sexual stimulation and Titration to the maximum tolerated dose) [9].

There are no data today with other drugs than sildenafil but it is estimated that similar causes of failure may exist depending of the different characteristics of the drugs (e.g. food interaction, duration of action).

Finally, clinician issues include patients who were misdiagnosed. These are patients with hypogonadism or hyperprolactinemia who need specific hormonal manipulation in order to cure erectile dysfunction, patients with Peyronie's disease who need treatment for penile curvature or pain during intercourse and patients who do not have erectile dysfunction but they experience ejaculatory dysfunction or sensory disturbances.

Patients and partners issues are currently recognized as extremely important in treatment failures. Some men may achieve an adequate erection for intercourse but discontinue the medication because they have unrealistic expectations, e.g. considering the drug as aphrodisiac. Lack of persistence is another cause of treatment failure. Patients need to know that if they respond to a drug, this response will be present in almost all of the times of intercourse attempts; occasional responses are not acceptable by most of the patients. Although no data exist on treatment reliability and patients' satisfaction, clinically a reliable response should be considered when $>75 \%$ of the attempts are successful. Patients may also experience fear about possible complications or side effects of the drugs despite clinicians' reassurance or may experience anxiety about new sexual life especially after long terms of abstinence due to erectile dysfunction. Finally patient issues include some cases where erectile function improved and spontaneous erections resumed and need no more treatment.

Partner issues are equally important and need to be addressed. These include women's sexual function issues such as pain, anorgasmia, vaginal dryness or lack of sexual interest, real or perceived sexual health 
concerns and general health status and comorbities. In conclusion, when a doctor simply prescribes a pill, he deals with only one aspect of a complex problem. Proper counselling and follow-up of the patients is necessary to overcome these issues.

\section{Identifying truly treatment failures}

In order to identify truly treatment failures, the physician need to address all the medication, clinician and patient/partner issues that presented before (Fig. 2). The probability of a misdiagnosis due to incomplete basic diagnostic evaluation from a non-expert physician must also be investigated [6]. A phychosexual evaluation should follow, including marital status and partner availability, relationship status (intimacy, anger, fear of separation), partner health status and comorbidities (pre- or post-menopause, diabetes mellitus, neurologic disease), partner sexual history (women sexual dysfunction) and possible psychiatric comorbidity. If possible, the partner should be also involved in the treatment plan. After proper counseling and instructions were given, the patient is encouraged for a re-trial of the oral drug for at least 4 attempts for intercourse with the new instructions on administration, as a recent study revealed inappropriate intake of sildenafil in $56 \%$ of treatment failures [10]. After adequate instructions on the proper use of sildenafil, one third of the initially non-responders to sildenafil converted to responders. The most easily reversible factor for treatment failure was the administration of sildenafil just before initiation of sexual activity followed by absence of sexual desire on sildenafil uptake and use of the drug with a full stomach. In this study, $12 \%$ of sildenafil failures were not aware that sexual stimulation was mandatory to achieve an erection, but $50 \%$ of them converted to responders when they were explained that sildenafil does not possess afrodisiac properties. Additionally, $63.6 \%$ of the patients who initially used the drug just before the initiation of sexual intercourse and $34.4 \%$ of those who took sildenafil with a full stomach responded to sildenafil with appropriate timing of administration.

These results confirmed by Atiemo et al. [11]. Incorrect administration accounted for $81 \%$ of sildenafil failures referred by primary care physicians. Reasons included administration after heavy meals, lack of sexual stimulation, short timing to intercourse and too few attempts for intercourse. The authors were

Identifications of truly non-responders to oral pharmacotherapy

Evaluation of drug administration

- Timing

- Dosing

- Sexual stimulation

- Number of attempts

- Food and drug interactions

Counselling / instructions on administration and re-trial (4 doses)

Trial with another PDE5 inhibitor (at least 4 attempts)

Fig. 2. Identification of truly non-responders to oral pharmacotherapy. 
able to salvage $41.5 \%$ of nonresponders after reeducation as measured by the General Assessment Question (GAQ) and Sexual Health Inventory for Men (SHIM) scores. Barada in an other study was able to salvage $54 \%$ of sildenafil due to similar reasons [12]. Vardi et al. presented data which show that proper instruction on sildenafil is essential for a high treatment success rate, especially in patients who had at least some initial response [13]. In this study, $78.4 \%$ of the physicians never mentioned food interaction with sildenafil intake and 54\% never mentioned the need for sexual stimulation. After proper instruction, erectile function domain scores increased significantly and overall $39.7 \%$ of the patients had an IIEF EF domain score above 20 .

A patient is considered as truly non-responder, if retrial of oral therapy after proper counseling and instruction administration fails.

\section{Treatment options for failures to oral pharmacotherapy}

\subsection{Switchover from one oral drug to another}

Treatment failure to oral pharmacotherapy means that the patient fails to respond to any oral drug for the treatment of erectile dysfunction. Today, we have 4 drugs available and many patients want to know if they will respond to another drug. Although data on apomorphine in sildenafil failures do not exist, apomorphine has limited efficacy compared to sildenafil and it is not expected to salvage sildenafil failures [14]. Tadalafil and vardenafil are the two new phosphodiesterase inhibitors. Due to the short time of availability, there are limited data on efficacy in sildenafil failures. Besides the lack of data in changing treatment from one oral drug to another, it is reasonable for the patient to consider a trial of all oral drugs available.

\subsection{Dosage adjustments}

Preliminary data show that higher doses of sildenafil (up to $200 \mathrm{mg}$ ) may salvage some patients who where sildenafil nonresponders. McMahon et al. concluded that sildenafil at doses of up to $200 \mathrm{mg}$ is an effective salvage therapy for $24.1 \%$ of previous sildenafil nonresponders but is limited by a significantly higher incidence of adverse effects and a $31 \%$ treatment discontinuation rate [15]. These data show that higher doses to salvage non-responders are not recommended, as no safety data are available and the side effects profile is not acceptable for a non life-threatening medical condition.

\section{Combination therapies}

\subsection{Drug combinations}

The rationale for combination therapies is widely known in medicine. Combining two or more drugs, the benefit of two different mechanisms of action may result in improved efficacy and may reduce side effects if the dose of each drug is lowered. Combination therapies are very common in medicine, particularly in hypertension treatment, but they have been used in the field of erectile dysfunction since the $80 \mathrm{~s}$ for intracavernosal injections (papaverine/phentolamine or papaverine/phentolamine/alprostadil) [16].

Following the approval of sildenafil, combination therapies have been used to salvage patients in whom monotherapy failed. The most popular approach has been the combination of sildenafil with intraurethral alprostadil. Nehra et al. were able to salvage patients who had undergone nerve-sparing prostatectomy by combination of $100 \mathrm{mg}$ of sildenafil and $500 \mu \mathrm{g}$ of MUSE [17]. All patients had failed $100 \mathrm{mg}$ sildenafil or $1000 \mu \mathrm{g}$ intraurethral alprostadil monotherapy. These patients did not want to try penile injection therapy and instead felt that the combination of sildenafil and intraurethral therapy improved their erections significantly, enabling them to achieve vaginal penetration. Follow-up of these patients for more than 24 months showed that nearly all of them were utilizing the combination for the desired effect. Similar results were presented by Mydlo et al. Combining sildenafil with intraurethral alprostadil, 60 out of 65 patients were satisfied and the erectile domain of the IIEF significantly improved [18].

The combination of sildenafil with intracavernosal injections is another possible combination, although there is lack of placebo-controlled trials. The intracavernosal injection program is the most efficacious pharmacotherapy available today and the aforementioned combination makes sense only in patients who do not respond to injections with the highest doses of the combination of papaverine, phentolamine and alprostadil (tri-mix solution), as data have shown that responders to high doses of tri-mix have limited possibilities to respond to sildenafil monotherapy [19]. McMahon et al. used sildenafil as salvage therapy in intracavernosal injection non-responders (alprostadil or tri-mix). They were able to salvage $31 \%$ of patients with combination of sildenafil with intracavernosal injection of tri-mix. However, combination therapy was associated with a $33 \%$ incidence of adverse effects, including a $20 \%$ incidence of dizziness side effects [19].

Experimental data on rats showed that apomorphine elicits a rise in intracavernous pressure that can be 
prolonged by sildenafil [20]. These results suggest that there may be a role for the combination of apomorphine and sildenafil in the management of erectile dysfunction. The combination of a central acting drug with a peripheral phosphodiesterase inhibitor is an attractive approach. Nonetheless, common adverse effects exist, such as flushing, hypotension and headache. No clinical data on such combination are available today.

Combination therapy of sildenafil with $4 \mathrm{mg}$ of doxazosin - an alpha adrenergic antagonist used for lower urinary tract symptoms proposed by De Rose et al., as this combination could salvage sildenafil nonresponders for the treatment of non-organic erectile dysfunction [21]. In another study, the addition of an oral alpha- blocker had a beneficial effect in patients with erectile dysfunction for whom intracavernosal therapy alone failed [22]. The potential role of alphablockade in synergy with other agents designed to treat erectile dysfunction however is contraindicated as safety studies clearly demonstrated that doxazosin has a synergistic hypotensive effect with all three phosphodiesterase inhibitors, while tamsulosin with sildenafil and vardenafil. No data are available for terazocin and alfuzosin.

The role of androgens in patients with ED and low testosterone levels (PADAM) has been recently under investigation. In one study, short-term testosterone administration increases testosterone and free testosterone levels and improves the erectile response to sildenafil likely by increasing arterial inflow to the penis during sexual stimulation [23]. In another study, sildenafil non responders with low testosterone levels were treated for 2 weeks with oral testosterone undecanoate. In $70 \%$ of them, the combination therapy of testosterone with sildenafil was successful by means of satisfactory erections for intercourse as measured by questions 3 and 4 of the IIEF [24]. Supplementation with androgens in sildenafil non-responders who are either significantly outside of the normal range or within the lower tertile or quartile may convert them to sildenafil responders; this therapeutic strategy is currently being evaluated in a number of prospective clinical trials.

\subsection{Psychosexual counselling}

Combination therapies may include other treatment modalities than drugs. Psychosexual counselling is an intriguing and important tool in the overall therapy and eventual success of treatment for both the patient and his partner. Counselling combined with a phosphodiesterase inhibitor seems to also offer an advantage over either method alone. Prospective data however, that provide an accurate assessment of the potential role of counselling in conjunction with other therapeutic measures are lacking.

\section{Vacuum erection devices}

Vacuum devices are considered a first line treatment option in erectile dysfunction. These devices have limited preference from patients, mainly because of the unnatural erection that they offer. However, after proper counselling and education they show high satisfaction rates among users especially in elderly patients with occasional intercourse attempts. In a preference study between sildenafil and vacuum devices in patients responded in the two treatments, $33.3 \%$ preferred vacuum devices due to the side effects of sildenafil while $66.6 \%$ preferred sildenafil due to fewer ejaculatory difficulties, efficacy, comfort and ease of use [25]. The vacuum erection device remains a preferred treatment option for a substantial number of patients with erectile dysfunction and can be offered in sildenafil failures.

\section{Intracavernosal injections}

Patients failing sildenafil are considered candidates for intracavernosal injection therapy as second-line treatment. Intracavernosal injections remain an excellent treatment option with proven efficacy and safety over time. Baniel et al. treated sildenafil non-responders after radical prostatectomy with intracavernosal injections of vasoactive drugs with $85 \%$ success rate [26]. Shabsigh et al. were able to salvage sildenafil non responders with intracavernosal injections of alprostadil. More than $85 \%$ improvement in questions 3 and 4 of the IIEF was recorded [27]. The tri-mix combination (papaverine, phentolamine, alprostadil) is certainly the more efficacious drug treatment for erectile dysfunction and can be used in severe cases of erectile dysfunction when intravernous alprostadil fails or it is not preferable due to side effects.

Another very interesting concept is the programmed administration of intracavernosal alprostadil in sildenafil non-responders. Gutierrez et al. provided $20 \mu \mathrm{g}$ intravernosal alprostadil every 2 weeks plus $50 \mathrm{mg}$ sildenafil or placebo on demand [28]. A clinically relevant improvement was observed in $60 \%$ of the patients on programmed intravernosal alprostadil and sildenafil, based on IIEF erectile function domain scores. In most of these patients the IIEF score changed from 'severe' to 'mild' erectile dysfunction. These 
preliminary data need further prospective trials to be confirmed.

\section{Penile prosthesis}

In patients who have failed oral therapy, intracavernosal injections or combination therapy, the implantation of a penile prosthesis remains the only treatment available. Three forms of penile prostheses are available (semi-rigid, malleable and inflatable) which vary in aesthetic and functional result, as well in acceptability and complications. The insertion of a penile prosthesis is an invasive and irreversible procedure, as well as expensive. Current modifications to penile prosthesis resulted in less mechanical malfunction rates and antibiotic coating reduces infections to less than $1 \%$ [29]. Penile implants still offer the best rate of success, over 95\%-inclusively partner's satisfaction-independent of etiology or the type of prosthesis used. The different types of devices must be presented to the patient prior to surgery, and he should decide what type of prosthesis he would like to have implanted, after being fully informed of the advantages and disadvantages of the individual types. Proper indication, careful patient selection, and sophisticated postoperative control not only of the mechanical aspects but also of the psychogenic impact is mandatory to fulfil the patient's and his partner's need of sexual satisfaction. However, the expectations of both the patient and his partner are often too high and both must learn to cope with the new situation.

\section{Management strategy for treatment failures}

Today, it is widely accepted that treatment approach follows a step-wise model. This approach divides existing treatments into first, second and third line therapies, based on several criteria: (1) ease of administration, (2) reversibility, (3) invasiveness and (4) cost [30]. This model is strongly recommended in the primary care setting. However, the medical condition and safety of the individual, as well as patient and partner preferences should be considered in all cases. First line therapies include oral drugs, vacuum erection devices and phychosexual counselling. Second and third line therapies are generally reserved for patients who show insufficient response to one or more first line therapies. Furthermore, the availability of many drugs and different treatment modalities raise interest in
Management strategy for non-responders to oral pharmacotherapy

Identify patient and partner needs and expectations

Informing patient about available treatment options

- Intracavernosal injections

- Combination therapies

- Penile prosthesis

Management strategy: shared decision making

Fig. 3. Management strategy for treatment failures.

combination therapies. Based on these concepts, a patient failing oral pharmacotherapy has to go on second line therapies that include intracavernosal injections and combination treatments. If he further fails second line therapies, the only option is a third line therapy that is implantation of a penile prosthesis.

The patient should be given every opportunity to choose among options, and to determine which fits best to his special needs and expectations. Patients' needs vary in their preference for information and involvement in the decision-making process, and for this reason the approach should always be individualised. Additionally, some patients may wish to consult with their partner or other health-care provider before selecting a specific management approach. These options should be respected and encouraged, if appropriate. It is important for the clinician not to assume an authoritarian or patriarchal role in the selection (or rejection) of specific treatment options. Instead, the clinician should aim to educate the patient as fully as possible, making full use of evidence-based literature and guidelines wherever possible, regarding the risks and benefits of each treatment. The clinician should also provide a supportive environment for shared decision-making. This management strategy (Fig. 3) must be supplemented by a careful followup in order to identify changes in patients' expectations, possible side effects that may need treatment optimization.

\section{Conclusions}

Most of the men with erectile dysfunction are successfully and safely treated with oral pharmacotherapy that included one of the three available phosphodiesterase 5 inhibitors: sildenafil, tadalafil and vardenafil. 
Apomorphine is another treatment option for oral pharmacotherapy but its efficacy is lower than phosphodiesterase inhibitors. Reasons for inappropriate use of oral drugs should be inspected. Androgen assessment and supplementation in the hypogonadal man is also an important consideration. In truly nonresponders cases, it is necessary to offer second and third line treatment alternatives. Second line treatments include intracavernosal injections of alprostadil and multidrug mixtures of vasoactive drugs (tri-mix).
Combination therapies are emerging and seem appealing, although prospective clinical trials are mandatory before their adaptation in physicians' armamentarium. When nonsurgical treatment options fail or are not satisfactory, penile prosthesis implantation may be the treatment that offers the best long-term solution for men with erectile dysfunction. Psychosexual counselling should always considered as an important addictive to every treatment strategy for the patient and his partner.

\section{References}

[1] Goldstein I, Lue TF, Padma-Nathan H, Rosen RC, Steers WD, Wicker PA. Oral sildenafil in the treatment of erectile dysfunction. Sildenafil Study Group. N Engl J Med 1998;338:1397-404.

[2] Wysowski DK, Swann J. Use of medications for erectile dysfunction in the United States, 1996 through 2001. J Urol 2003;169:1040-2.

[3] Hatzichristou DG, Apostolidis A, Tzortzis V, Ioannides E, Yannakoyorgos K, Kalinderis A. Sildenafil versus intracavernous injection therapy: efficacy and preference in patients on intracavernous injection for more than 1 year. J Urol 2000;164:1197-200.

[4] Hellstrom WJ, Gittelman M, Karlin G, Segerson T, Thibonnier M, Taylor T, et al. Sustained efficacy and tolerability of vardenafil, a highly potent selective phosphodiesterase type 5 inhibitor, in men with erectile dysfunction: results of a randomized, double-blind, 26week placebo-controlled pivotal trial. Urology 2003;61:8-14.

[5] Brock GB, McMahon CG, Chen KK, Costigan T, Shen W, Watkins $\mathrm{V}$, et al. Efficacy and safety of tadalafil for the treatment of erectile dysfunction: results of integrated analyses. J Urol 2002;168:1332-6.

[6] Hatzichristou D, Hatzimouratidis K, Bekas M, Apostolidis A, Tzortzis V, Yannakoyorgos K. Diagnostic steps in the evaluation of patients with erectile dysfunction. J Urol 2002;168:615-20.

[7] Rutchik SD, Baudiere M, Wade M, Sullivan G, Rayford W, Goodman J. Practice patterns in the diagnosis and treatment of erectile dysfunction among family practice physicians. Urology 2001; 57:146-50.

[8] McCullough AR, Barada JH, Fawzy A, Guay AT, Hatzichristou D. Achieving treatment optimization with sildenafil citrate (Viagra) in patients with erectile dysfunction. Urology 2002;60:28-38.

[9] Hatzichristou DG. Sildenafil citrate: lessons learned from 3 years of clinical experience. Int J Impot Res 2002;14(Suppl 1):S43-52.

[10] Hatzichristou D, Apostolidis A, Bekos A, Tzortzis V, Ioannides E, Yannakoyorgos K. Sildenafil failures may be due to inadequate instructions and follow-up: a study on 100 non-responders. Int J Impot Res 2001;13:S32 [Abstract 85].

[11] Atiemo HO, Szostak MJ, Sklar GN. Salvage of sildenafil failures referred from primary care physicians. J Urol 2003;170:2356-8.

[12] Barada J. Salvage of 'sildenafil (viagra) failures': benefits of patient and retreatment with sildenafil. Int J Impot Res 2001;13:S49 [Abstract 137].

[13] Vardi Y, Chen J, Sheinfeld O, Richter S, Raviv G, Grunwald I. A multicenter study, evaluating the effect of instruction and re-dosing of sildenafil 'failures'. Int J Impot Res 2002;14:S15 [Abstract PS-14-12].

[14] Strebel RT, Reitz A, Tenti G, Curt A, Hauri D, Schurch B. Apomorphine sublingual as primary or secondary treatment for erectile dysfunction in patients with spinal cord injury. BJU Int 2004;93:100-4.

[15] McMahon CG. High dose sildenafil citrate as a salvage therapy for severe erectile dysfunction. Int J Impot Res 2002;14:533-8.

[16] Steers WD. Viability and safety of combination drug therapies for erectile dysfunction. J Urol 2003;170:S20-3.
[17] Nehra A, Blute ML, Barrett DM, Moreland RB. Rationale for combination therapy of intraurethral prostaglandin $\mathrm{E}(1)$ and sildenafil in the salvage of erectile dysfunction patients desiring noninvasive therapy. Int J Impot Res 2002;14(Suppl 1):S38-42.

[18] Mydlo JH, Volpe MA, Macchia RJ. Initial results utilizing combination therapy for patients with a suboptimal response to either alprostadil or sildenafil monotherapy. Eur Urol 2000;38:30-4.

[19] McMahon CG, Samali R, Johnson H. Treatment of intracorporeal injection nonresponse with sildenafil alone or in combination with triple agent intracorporeal injection therapy. J Urol 1999;162: 1992-7.

[20] Andersson KE, Gemalmaz H, Waldeck K, Chapman TN, Tuttle JB, Steers WD. The effect of sildenafil on apomorphine-evoked increases in intracavernous pressure in the awake rat. J Urol 1999;161: 1707-12.

[21] De Rose AF, Giglio M, Traverso P, Lantieri P, Carmignani G. Combined oral therapy with sildenafil and doxazosin for the treatment of non-organic erectile dysfunction refractory to sildenafil monotherapy. Int J Impot Res 2002;14:50-3.

[22] Kaplan SA, Reis RB, Kohn IJ, Shabsigh R, Te AE. Combination therapy using oral alpha-blockers and intracavernosal injection in men with erectile dysfunction. Urology 1998;52:739-43.

[23] Aversa A, Isidori AM, Spera G, Lenzi A, Fabbri A. Androgens improve cavernous vasodilation and response to sildenafil in patients with erectile dysfunction. Clin Endocrinol (Oxf) 2003;58: 632-8.

[24] Kalinchenko SY, Kozlov GI, Gontcharov NP, Katsiya GV. Oral testosterone undecanoate reverses erectile dysfunction associated with diabetes mellitus in patients failing on sildenafil citrate therapy alone. Aging Male 2003;6:94-9.

[25] Chen J, Mabjeesh NJ, Greenstein A. Sildenafil versus the vacuum erection device: patient preference. J Urol 2001;166:1779-81.

[26] Baniel J, Israilov S, Segenreich E, Livne PM. Comparative evaluation of treatments for erectile dysfunction in patients with prostate cancer after radical retropubic prostatectomy. BJU Int 2001; 88:58-62.

[27] Shabsigh R, Padma-Nathan H, Gittleman M, McMurray J, Kaufman $\mathrm{J}$, Goldstein I. Intracavernous alprostadil alfadex (EDEX/VIRIDAL) is effective and safe in patients with erectile dysfunction after failing sildenafil (Viagra). Urology 2000;55:477-80.

[28] Gutierrez P, Hernandez P, Jimenez A, Mas M. Combining programmed intracavernous PGE1 injections and sildenafil on demand to salvage sildenafil nonresponders. Int J Impot Res 2002;14:S37 [Abstract S33.05].

[29] Carson CC. Diagnosis, treatment and prevention of penile prosthesis infection. Int J Impot Res 2003;15(Suppl 5):S139-46.

[30] The process of care model for evaluation and treatment of erectile dysfunction. The Process of Care Consensus Panel. Int J Impot Res 1999;11:59-70. 


\section{CME questions}

Please visit http://www.uroweb.org/updateseries to answer these CME questions on-line. The CME credits will then be attributed automatically.

1. One of your patients comes to your office after a PDE5 inhibitor trial, complaining for inadequate response after 2 sexual attempts. What do you suggest?

A. Try a different oral drug.

B. Try intracavernosal injections.

C. Give adequate instructions and retrial.

D. Refer to a sexologist.

2. A patient is truly non-responder after adequate instructions. What do you propose to him?

A. Intracavernosal injections.

B. Penile prosthesis implantation.

C. Vacuum device.

D. Presentation of all treatment options and shared decision-making.
3. A patient comes back to your office after successful trial of an oral drug requesting different treatment because his partner refused the use of an oral drug due to safety reasons. What do you advise him?

A. Offer him an alternative treatment.

B. Refer the couple to a cardiologist.

C. Refer the couple to a sexologist.

D. Offer information and education to the couple.

4. A patient with free medical history and psychogenic erectile dysfunction, comes to your office asking about the most efficacious and safe PDE5 inhibitor. What do you propose to him?

A. Sildenafil.

B. Tadalafil.

C. Vardenafil.

D. Explain to him that all PDE5 inhibitors have a proven efficacy and safety profile. 